TITLE:

\title{
Time evolution of the electron diffusion region and the reconnection rate in fully kinetic and large system
}

\author{
AUTHOR(S): \\ Fujimoto, K
}

\section{CITATION:}

Fujimoto, K. Time evolution of the electron diffusion region and the reconnection rate in fully kinetic and large system. PHYSICS OF PLASMAS 2006, 13(7): 072904.

\section{ISSUE DATE: \\ 2006-07 \\ URL: \\ http://hdl.handle.net/2433/50016 \\ RIGHT:}

Copyright 2006 American Institute of Physics. This article may be downloaded for personal use only. Any other use requires prior permission of the author and the American Institute of Physics. 


\title{
Time evolution of the electron diffusion region and the reconnection rate in fully kinetic and large system
}

\author{
Keizo Fujimoto ${ }^{\text {a) }}$ \\ Department of Geophysics, Graduate School of Science, Kyoto University, Kitashirakawa Oiwaketyo, \\ Sakyo-ku, Kyoto 606-8502, Japan
}

(Received 6 February 2006; accepted 30 May 2006; published online 21 July 2006)

\begin{abstract}
Time evolutions of the electron diffusion region embedded in the ion-scale diffusion region and the reconnection rate associated with magnetic reconnection are investigated using 2-1/2 dimensional full kinetic simulations in a large system, so that any effects of the downstream boundary conditions are negligible. The simulation code employs the adaptive mesh refinement technique and the particle splitting algorithm to the conventional particle-in-cell code, which enable us to perform large-scale full particle simulations. It is shown that the reconnection rate increases associated with magnetic reconnection and reaches a peak value large enough for fast reconnection, but then it decreases as time goes on, even though the effects of the system boundary are negligible. The key process responsible for slowing the reconnection processes is the extension of the electron diffusion region in association with the enhancement of the polarization electric field directing toward the neutral sheet in the electron inflow region. The polarization electric field is caused by the inertia difference between ions and electrons, and enhanced by the meandering motions of the background ions. In order to confirm the role of the polarization electric field, we compare the simulation results with $m_{i} / m_{e}=1$ and 100. It is found that (1) a quasi-steady reconnection is achieved in the system where the polarization electric field does not arise, (2) a large reconnection rate is obtained, even in the system without the Hall effects. It is suggested that the anomalous resistivity due to the Buneman-type instability might be required to support a steady-state fast reconnection.
\end{abstract}

(C) 2006 American Institute of Physics. [DOI: 10.1063/1.2220534]

\section{INTRODUCTION}

Magnetic reconnection is widely believed to play an important role in the magnetospheric substorm and solar flares as a fast conversion process of the magnetic energy to plasma kinetic and thermal energies. In reconnection processes, the ideal magnetohydrodynamic (MHD) condition breaks down in the diffusion region arising around a magnetic $\mathrm{X}$ line, where dissipation processes of the magnetic field are significant. ${ }^{1}$ In a sufficiently collisional plasma, the resistive MHD theory ${ }^{2-4}$ is valid for describing the diffusion region by parametrizing effects of the classical Coulomb collisions. ${ }^{5}$ However, in the collisionless plasma as seen in the Earth's magnetosphere, the magnetic diffusion due to the classical resistivity is too small to drive fast reconnection. In such a system, we need to replace the standard Ohm's law in the resistive MHD by the generalized Ohm's law, written as

$$
\boldsymbol{E}+\boldsymbol{V} \times \boldsymbol{B}=-\frac{m_{e}}{e} \frac{d \boldsymbol{V}_{e}}{d t}-\frac{1}{n e} \boldsymbol{\nabla} \cdot \boldsymbol{P}_{e}+\frac{1}{n e} \boldsymbol{J} \times \boldsymbol{B},
$$

where $\boldsymbol{E}$ is the electric field, $\boldsymbol{B}$ the magnetic field, $\boldsymbol{J}$ the current density, $n$ the plasma number density, $\boldsymbol{V}_{e}$ the electron flow velocity, $\boldsymbol{P}_{e}$ the electron pressure tensor, and $m_{e}$ the electron mass. The first and second terms on the right-hand side arise from electron inertial effects, and the scale sizes are evaluated as the electron inertial length $\lambda_{e}$ and the electron gyroradius $\lambda_{g e}$, respectively. The last term represents the

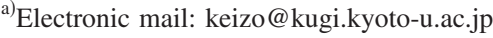

decoupling between ions and electrons, so-called Hall effects, and has a scale size of the ion inertial length $\lambda_{i}$. When the scale length of gradient is much larger than any of these characteristic lengths, the frozen-in condition holds.

In a two-fluid system consisting of ions and electrons, the inflowing ions can be decoupled from the ambient magnetic field when they approach the neutral sheet as close as $\lambda_{i}$. Thus the region within $\lambda_{i}$ is the diffusion region where the frozen-in condition breaks down. Electrons, however, still remain magnetized at this distance, since their inertia is much smaller than those of ions. Instead, their frozen-in constraint can break down at the distance comparable with the local gyroradius $\lambda_{g e}$, within which electrons are expected to perform the meandering motions (e.g., Ref. 6). This nonmagnetized region for electrons is called the electron diffusion region. Therefore the diffusion region in the two-fluid system develops a two-scale structure, embedding the electron diffusion region within the ion-scale diffusion region.

Inside the diffusion region but outside the electron diffusion region, ions are decoupled from the ambient magnetic field, but electrons are still frozen in to the field and continue to move with the $\boldsymbol{E} \times \boldsymbol{B}$ drift motion toward the current sheet. This relative motion between ions and electrons produces currents in the vicinity of the magnetic $\mathrm{X}$ line, forming the Hall current system. ${ }^{7,8}$ On the other hand, the kinetic behavior of electrons becomes important in the electron diffusion region. It has been revealed by using two-dimensional kinetic simulations that the dissipation process in the electron diffusion region is supported by the electron inertial term and 
the gradient term of the electron pressure tensor in the generalized Ohm's law (1). ${ }^{9-13}$ Though reconnecting of the magnetic field actually proceeds in this region, it has been suggested that the electron dynamics should have little or no effect on the reconnection rate. ${ }^{14,15}$ Instead, ion dynamics can control the reconnection processes, that is, the electronscale structure. They conclude that a high reconnection rate results from the Hall term in the generalized Ohm's law (1), so that the inclusion of the Hall effects is a sufficient condition to achieve fast reconnection. This indicates that threedimensional effects arising along the current sheet would not be essential for obtaining the high reconnection rate. ${ }^{16}$ Though recent simulation studies comparing MHD, Hall MHD, hybrid, and full particle simulations confirmed the importance of the Hall effects, the system did not reach steady state and the reconnection rate decreased after it reached a peak value. ${ }^{17-20}$ They attributed the depression to the periodic system in the direction of the initial magnetic field, in which compressional effects in the magnetic islands could affect the reconnection processes. One of the solutions in order to suppress the boundary effects is to perform the simulations in a large system so that the periodicity is negligible therein.

Such large-scale simulations including the Hall effects were examined using a two-dimensional hybrid $\operatorname{code}^{21}$ and a two-dimensional two-fluid code. ${ }^{22}$ Both results also indicate the importance of the Hall effects for fast reconnection and show a quasi-steady reconnection with the reconnection rate of the order of $0.1 V_{\mathrm{A} 0} B_{0}$, where $V_{\mathrm{A} 0}$ and $B_{0}$ are, respectively, the Alfvén velocity and the magnetic field defined in the asymptotic lobe region, and the reconnection rate is estimated by the electric field strength along the $\mathrm{X}$ line. However, Karimabadi et al. ${ }^{23}$ recently compared two different hybrid simulations with and without the Hall term under an open boundary condition, and demonstrated that ion kinetic behavior alone could give rise to a fast reconnection, even in the absence of the Hall effects. Thus, the Hall effects may not be necessarily required for fast reconnection.

In the present work we shows result from 2-1/2 dimensional full particle simulations in a large system, in which both ions and electrons are treated as superparticles. Our purpose in this study is to see a time evolution of the diffusion region in a fully kinetic system without the complicated effects due to the periodic boundaries. The main concern is how the ion dynamics affects the electron-scale structure, that is, the reconnection processes. It is still difficult to conduct large-scale simulations using conventional particle-incell (PIC) codes because of limited computer resources. Thus, we employed the adaptive mesh refinement (AMR) technique and the particle splitting algorithm to the conventional PIC code. ${ }^{24}$ The adoption of these techniques enables us to perform effectively high-resolution simulations including kinetic processes of magnetic reconnection.

\section{SIMULATION MODEL}

In the present study we employ a 2-1/2 dimensional electromagnetic PIC code with the AMR technique and the particle splitting algorithm, which has been described by
Fujimoto and Machida. ${ }^{24}$ The AMR technique subdivides and removes computing cells dynamically in accordance with a refinement criterion and quite effective to achieve high-resolution simulations of phenomena that locally include microscale processes. In the present code, the spatial resolution is increased by introducing finer cells hierarchically onto the uniform base cells that cover the entire simulation area. If a base cell is refined, four child cells that have half the size of the base cell are generated. These child cells can be also refined in turn and finer cells are produced, and so on. One of the main problems in developing the electromagnetic PIC codes using the AMR technique is the decrease in the number of particles per cell in the refined region. In order to solve this problem, we subdivide particles residing in the subdivided cells and control the number of particles per cell. We employ the particle splitting algorithm developed by Lapenta, ${ }^{25}$ in which the moments on each grid (the charge and current densities), the total charge, mass, momentum, and energy of particles, and the distribution function of particles are retained between before and after the particle splitting. Each cell is needed to have information of the parent, child, and neighboring cells, and the particles residing in the cell. The communication between cells or between a cell and the particles in the cell is supported by a set of pointers, constructing the fully threaded tree structure. ${ }^{26}$ A refinement level $L$ in the hierarchical cell structure is defined by using the cell size of the level $\left(\Delta_{L}\right)$ as $L \equiv \log _{2}\left(l_{z} / \Delta_{L}\right)$, where $l_{z}$ is the vertical size of the two-dimensional simulation area. We use only cells with integer level. A more detailed description on our code is shown in Fujimoto and Machinda. ${ }^{24}$

The refinement condition in the current study is defined by three physical values. The first is the local electron Debye length, $\lambda_{\mathrm{D} e}=v_{\mathrm{th}, e} / \sqrt{2} \omega_{p e}$, which is required to avoid a numerical heating of local plasma, where $v_{\mathrm{th}, e}=\sqrt{2 T_{e} / m_{e}}$ is the electron thermal velocity $\left(T_{e}\right.$ and $m_{e}$ are the temperature and mass of electrons, respectively), and $\omega_{p e}$ is the electron plasma frequency. We use the initial value of $v_{\text {th }, e}$ for calculation of $\lambda_{\mathrm{D} e}$, because electrons are expected to be heated in the region where the electron dynamics is important, that is, we use the minimum value of $\lambda_{\mathrm{D} e}$. The second is the out-ofplane electron flow velocity $\left(V_{e y}\right)$, because the inertial term (the first term of the right-hand side) in the generalized Ohm's law (1) becomes dominant in the region where the electron flow is strong, so that the dissipation of the magnetic field becomes strong and higher resolution is required. The third is the in-plane electron current density $\left(J_{\text {exz }}\right.$ $\left.=\sqrt{J_{e x}^{2}+J_{e z}^{2}}\right)$, which is required because intense currents can excite microinstabilities so that electron-scale waves are expected to arise. In each time step, if $\lambda_{\mathrm{D} e}, V_{e y}$, and $J_{e x z}$ calculated at the center of a cell satisfy the condition, $\Delta_{L}$ $>2.0 \lambda_{\mathrm{D} e}$ or $V_{e y}>2.0 V_{\mathrm{A}}$ or $J_{e x z}>0.5 e n_{\mathrm{ps}} V_{\mathrm{A}}$, the cell is subdivided and four child cells are produced, otherwise, the child cells, if any, are removed. Here, $V_{\mathrm{A}}$ is the Alfvén velocity defined by the initial lobe field $\left(B_{0}\right)$ and plasma sheet density $\left(n_{\mathrm{ps}}\right)$, and $e$ is the electron charge. The particle splitting is also performed in each time step, but this is done only for the background particles whose initial distribution will be described later. We carry out not only particle splitting but 
also particle coalescence in order to reduce the number of particles in the unrefined region. The particle coalescence algorithm is implemented once in a few hundred time steps for the background particles. In both the particle splitting and coalescence algorithms, we never choose particles in the most refined (finest) cells to avoid numerical errors in physically important regions. In the present study, the hierarchical cell structure consists of four cell layers.

The initial magnetic field configuration is given as $B_{x}(z)=-B_{0} \tanh (z / \lambda)$, and the corresponding density is $n(z)$ $=n_{\mathrm{ps}} \operatorname{sech}^{2}(z / \lambda)$, where $\lambda$ is the half width of the initial plasma sheet and set as $\lambda=0.5 \lambda_{i 0}\left(\lambda_{i 0}\right.$ is the ion inertial length defined by $\left.n_{\mathrm{ps}}\right)$. In addition to the equilibrium, a small perturbation is superposed in the form

$$
\begin{aligned}
B_{x p}(x, z)= & 2 a_{0} / \lambda \operatorname{sech}^{2}\left[\left(x-l_{x} / 2\right) / L^{\prime}\right] \operatorname{sech}^{2}(z / \lambda) \tanh (z / \lambda), \\
B_{z p}(x, z)= & -2 a_{0} / L^{\prime} \operatorname{sech}^{2}\left[\left(x-l_{x} / 2\right) / L^{\prime}\right] \operatorname{sech}^{2}(z / \lambda) \\
& \times \tanh \left[\left(x-l_{x} / 2\right) / L^{\prime}\right],
\end{aligned}
$$

where $l_{x}$ is the horizontal length of the simulation area, and $a_{0}$ and $L^{\prime}$ provide the amplitude and horizontal size of the perturbation, respectively. In the present study, $a_{0}$ $=0.15 B_{0} \lambda_{i 0}, L^{\prime}=3.8 \lambda_{i 0}$, and $l_{x} \times l_{z}=123 \lambda_{i 0} \times 30.7 \lambda_{i 0}$ are chosen, unless otherwise mentioned. Furthermore, the background plasma is loaded as $n_{b}(z)=n_{b 0} \tanh ^{2}(z / \lambda)$ and $n_{b 0}$ $=0.044 n_{\mathrm{ps}}$ in order to describe the lobe plasma in the magnetospheric tail. Although there appears a weak pressure imbalance due to this background profile, it is quickly justified without any significant modification of the current sheet structure. Once the simulation starts, the initial plasma sheet density and current profiles are quickly modified to adjust the field perturbation. As a result, a thinner current sheet is formed near the center of the simulation area $\left(x=l_{x} / 2, z=0\right)$, so that the tearing instability selectively develops therein. The cell size is $\Delta_{L_{B}}=0.12 \lambda_{i 0}$ for the coarsest cells and $\Delta_{L_{D}}$ $=0.015 \lambda_{i 0}$ for the finest cells, and the time step is $\Delta t \omega_{c i}$ $=8.0 \times 10^{-4}$ for all particles and refined regions in order to satisfy the Courant condition on the finest cells, where $\omega_{c i}$ is the ion cyclotron frequency defined by the lobe field $\left(B_{0}\right)$. The initial plasma condition is $m_{i} / m_{e}=100, T_{i, \mathrm{ps}} / T_{e, \mathrm{ps}}=8.0$, $T_{i, \text { lobe }} / T_{e, \text { lobe }}=1.0, T_{e, \text { lobe }} / T_{e, \mathrm{ps}}=1.0$, unless otherwise mentioned, and $c / V_{\mathrm{A}}=16.7$, where $T_{s, \mathrm{ps}}$ and $T_{s, \mathrm{lobe}}$ are the temperatures of the species $s$ at the central plasma sheet and the magnetic lobe, respectively, and $c$ is the velocity of light. We assume the periodic boundary in the $x$ direction and the conducting wall in the $z$ direction.

\section{RESULTS}

\section{A. Time evolution of the reconnection rate}

The evolution of the reconnected magnetic flux, $\psi$ $=\int_{0}^{l_{x} / 2}\left|B_{z}(x, z=0)\right| d x$, is plotted in Fig. 1(a). Here, it is assumed that the $\mathrm{X}$ line is formed at the center of the simulation area $\left(x=l_{x} / 2, z=0\right)$. It increases very slowly in the first half of the simulation time, but develops at a drastically high rate after $t \omega_{c i} \approx 12.5$, so that fast reconnection is switched on. The reconnection rate should be given by the instantaneous growth rate of $\psi$, which is rigorously derived from Faraday's
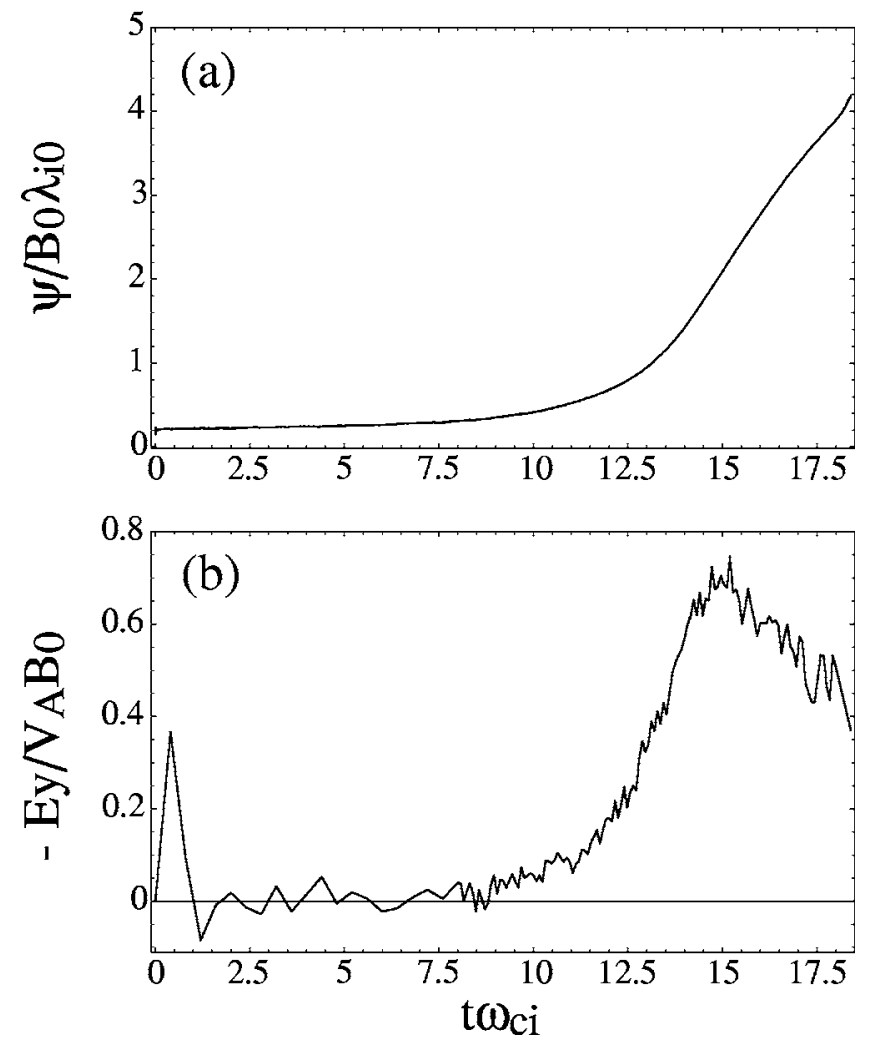

FIG. 1. Time evolutions of (a) the reconnected magnetic flux, (b) the outof-plane electric field at the center of the simulation area $\left(x=l_{x} / 2, z=0\right)$.

law as $\partial \psi / \partial t=-E_{y}\left(x=l_{x} / 2, z=0\right)$, which expresses the electric field imposed along the $\mathrm{X}$ line. Figure 1(b) shows the time evolution of the out-of-plane electric field at the center of the simulation area. The reconnection rate develops associated with the evolution of magnetic reconnection until it reaches a peak at $t \omega_{c i} \approx 15$, and a fast reconnection with $\left|E_{y}\right| \sim 0.7 V_{\mathrm{A}} B_{0}=0.15 V_{\mathrm{A} 0} B_{0}$ is achieved, where $V_{\mathrm{A} 0}$ is the Alfvén velocity in the asymptotic lobe region (defined by $B_{0}$ and $\left.n_{b 0}\right)$. However, after then it starts to decrease and fall to nearly the half of the peak value at the end of the simulation. This time history is similar to those obtained in other particle simulations having smaller simulation areas, in which the periodicity of the system has tended to affect the reconnection rate and slow down the reconnection processes. ${ }^{17-20} \mathrm{In}$ the present run, however, the simulation area is much larger than in the previous runs, so that it is expected that the effects of the periodic boundaries should be significantly reduced. In order to check the effects of the periodicity, we compare the runs having a different size of the simulation area in the $x$ direction. Figure 2 shows the time profiles of the reconnection rate obtained in five different system sizes in the $x$ direction. In the smaller systems $\left(l_{x}=15.4 \lambda_{i 0}\right.$ and $\left.30.7 \lambda_{i 0}\right)$, as employed in previous particle simulations, the onsets of the fast reconnection are delayed compared with those in the larger systems. This is because the magnetic tension force accelerating plasma away from the $\mathrm{X}$ line is opposed by a total pressure (the plasma pressure + the magnetic pressure) gradient in the downstream region of the $\mathrm{X}$ line. ${ }^{22}$ Thus, the profiles for the smaller systems demonstrate that the periodic boundaries already affect the reconnection 


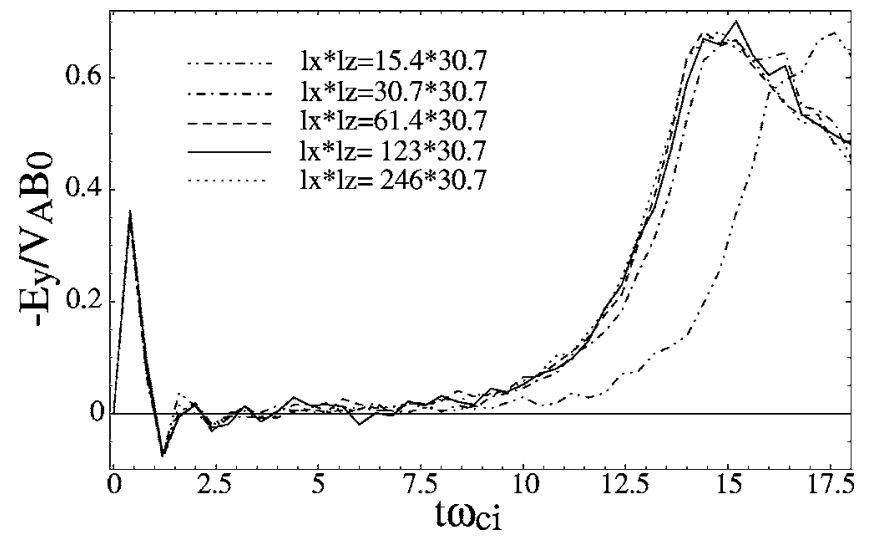

FIG. 2. A comparison of the time evolutions of the out-of-plane electric field at the center of the simulation area (the reconnection rate). Five system sizes are examined: $l_{x} \times l_{z}=15.4 \lambda_{i 0} \times 30.7 \lambda_{i 0}$ (dashed double-dotted line), $30.7 \lambda_{i 0} \times 30.7 \lambda_{i 0}$ (dashed-dotted line), $61.4 \lambda_{i 0} \times 30.7 \lambda_{i 0}$ (dashed line), $123 \lambda_{i 0} \times 30.7 \lambda_{i 0}$ (solid line), and $246 \lambda_{i 0} \times 30.7 \lambda_{i 0}$ (dotted line). The results of $l_{x} \geq 61.4$ are in good agreement with each other, which indicates that the effects of the periodic boundaries are mostly negligible in these runs.

processes in the early phase. On the other hand, the results of the larger systems $\left(l_{x}=61.4 \lambda_{i 0}, 123 \lambda_{i 0}\right.$, and $\left.246 \lambda_{i 0}\right)$ are in good agreement with each other, which indicates that the periodicity of the system is mostly negligible and the evolution of the reconnection rate is independent of the system size in these runs. Therefore the depression of the reconnection rate after $t \omega_{c i} \approx 15$ in the present run $\left(l_{x} \times l_{z}=123 \lambda_{i 0}\right.$ $\left.\times 30.7 \lambda_{i 0}\right)$ is not due to the periodic system but rather due to spontaneous effects essentially inherent in the reconnection processes.

\section{B. Structure change around the electron diffusion region}

The density profiles along the $z$ direction at $x=l_{x} / 2$ are shown in Fig. 3(a) for ions (red solid lines) and electrons (blue solid lines) at two different times: $t \omega_{c i}=12.8$ (left) and 16.7 (right). At $t \omega_{c i}=12.8$, both the ion and electron density profiles have peaks at $z=0$ that are reminiscent of the initial equilibrium profiles. On the other hand, at $t \omega_{c i}=16.7$, electrons have still a peak at $z=0$ while ion peaks appear at the flanks of the electron peak and a density hole is formed at $z=0$. The double-peaked structure in the ion density profile, as seen at $t \omega_{c i}=16.7$, indicates that most ions carry out meandering motions near the $\mathrm{X}$ line, staying long around the turning points and passing fast around $z=0$. The red dashed lines in Fig. 3(a) denote the density profiles of the background cold ions, which are initially loaded only in the lobe region. Its profile at $t \omega_{c i}=12.8$ is not yet changed from the initial profile, in essence. However, as magnetic reconnection proceeds, the background ions approach the $\mathrm{X}$ line and start the meandering motions. It is found that the double-peaked structure at $t \omega_{c i}=16.7$ is mainly produced by the background ions meandering around the $\mathrm{X}$ line. Because the hot ions initially loaded in the current sheet have large velocity in the out-of-plane direction in order to produce the current supporting the initial magnetic field profile, they can easily escape from the diffusion region due to the Lorentz force before their meandering motions have dominant. Thus, as the density of the background cold ions dominates that of the plasma sheet ions in the vicinity of the $\mathrm{X}$ line, the doublepeaked structure becomes dominant. This structure change occurs around $t \omega_{c i}=15$ in the present run.

The difference in the density peak position between ions and electrons after $t \omega_{c i} \approx 15$ are mainly caused by their inertia difference, and give rise to a strong polarization electric field $E_{z}$ directing toward the neutral sheet in the electron inflow region. Its profiles along the $z$ direction are shown in Fig. 3(b), and the two-dimensional snapshots are also given in Fig. 4. Note that the polarization electric field $E_{z}$ already appears at $t \omega_{c i}=12.8$ near the $\mathrm{X}$ line, though the ion meandering motions are not yet significant. By this time, electrons have been confined within a much thinner current sheet than the initial one, while ions still maintain the broad current sheet with the initial width. Thus the electron density slightly exceeds the ion density near $z=0$ [Fig. 3(a)], which gives rise to the polarization electric field (e.g., Refs. 27-31). The polarization electric field is enhanced and its imposed region is broadened not only in the $z$ direction but also in the $x$ direction, associated with the meandering motions of the background ions [Figs. 3(b) and 4]. The time evolution of the electric field directing toward the neutral sheet $\overline{E_{z}}=-z /|z| E_{z}$, averaged over the electron inflow region $\left(l_{x} / 2-0.2 \lambda_{i 0} \leq x\right.$ $\left.\leq l_{x} / 2+0.2 \lambda_{i 0}, 0.25 \lambda_{i 0} \leq|z| \leq 0.5 \lambda_{i 0}\right)$, is plotted in Fig. 5. $\overline{E_{z}}$ develops after $t \omega_{c i} \approx 13$ as magnetic reconnection proceeds. Especially, its growth rate is enhanced after $t \omega_{c i} \approx 15$, indi-
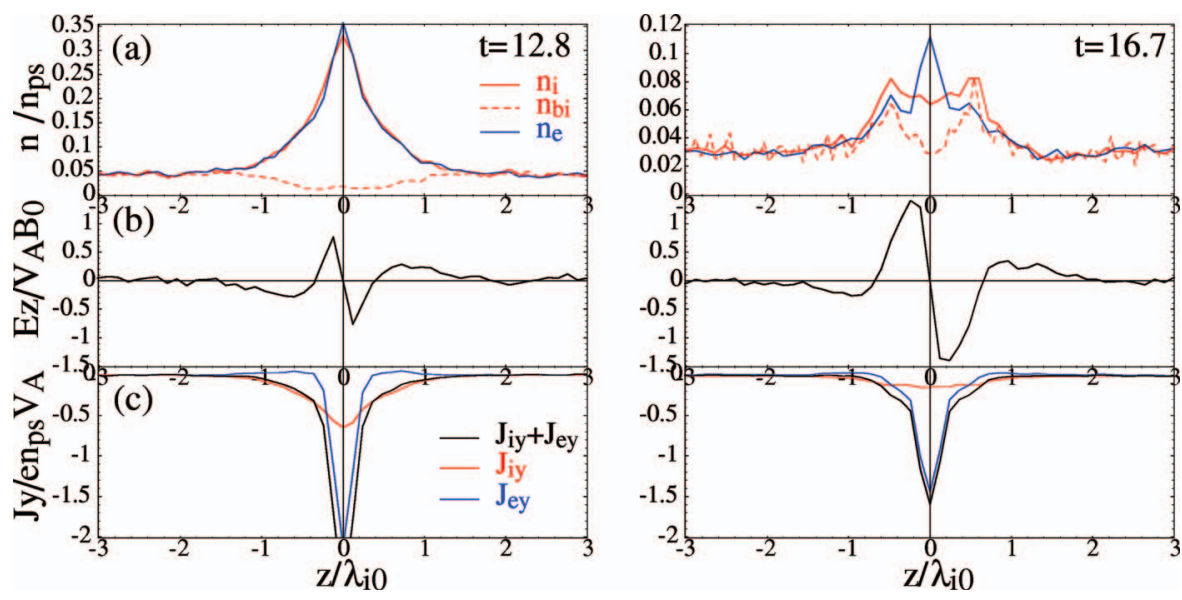

FIG. 3. (Color) A comparison of structures along the $z$ direction through the center of the simulation area $\left(x=l_{x} / 2\right.$, $z=0)$ at two different times: $t \omega_{c i}$ $=12.8$ (left) and 16.7 (right). (a) The number densities of ions (red solid lines) and electrons (blue solid lines). The red dashed lines represent the contribution from the background cold ions. (b) The electric field along the $z$ direction. (c) The out-of-plane current densities (black solid lines), which are calculated by summing up the ion and electron current densities (red and blue lines, respectively). 


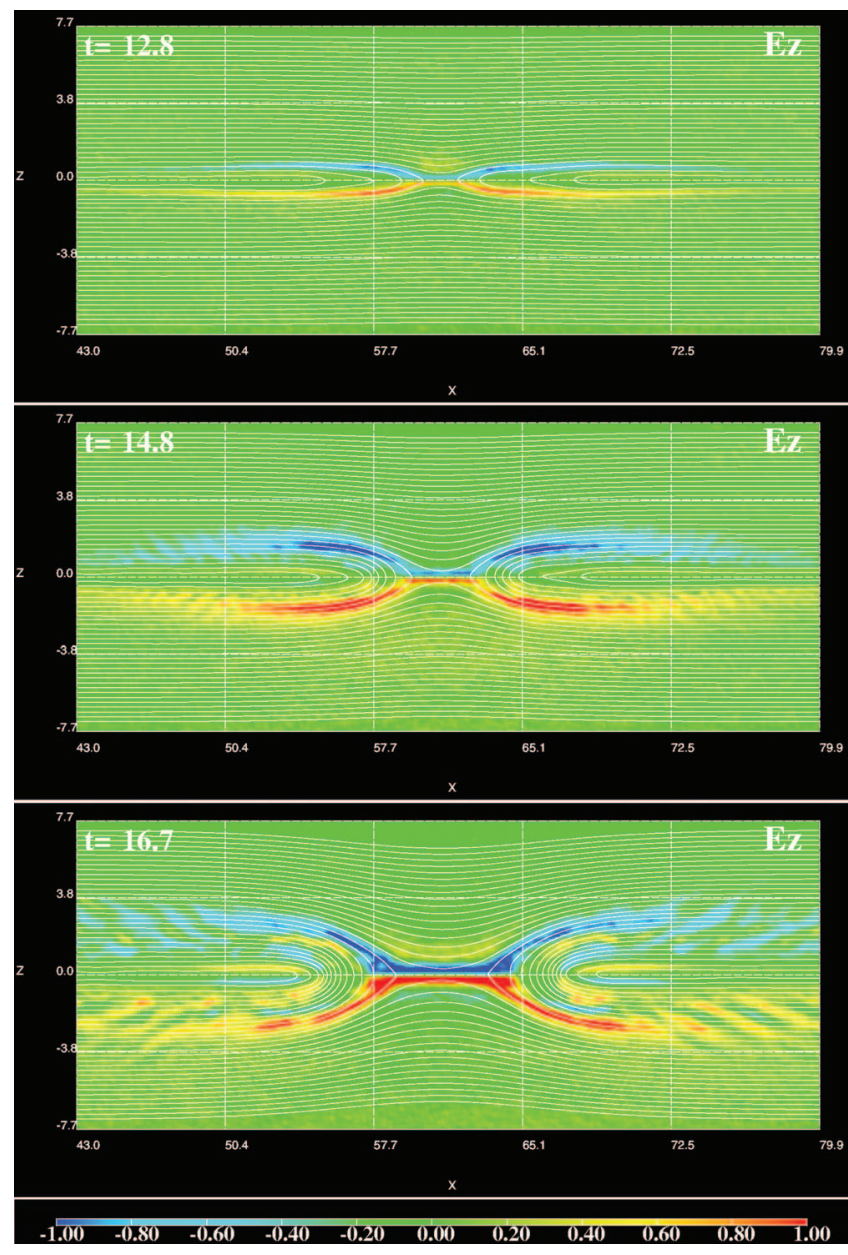

FIG. 4. (Color) Snapshots of the electric field along the $z$ direction at $t \omega_{c i}$ $=12.8,14.8$, and 16.7. Magnetic field lines (white solid lines) are superposed on each panel.

cating that the background ions carrying out the meandering motions become dominant around the $\mathrm{X}$ line. Figure 3(c) shows the comparison of the out-of-plane current density profiles (black lines), which are calculated by summing up the ion and electron current densities (denoted by red and blue lines, respectively). It is clearly shown that the polarization electric field $E_{z}$ forces the inflow electrons to do the strong $\boldsymbol{E} \times \boldsymbol{B}$ drift toward the out-of-plane direction and enhances the out-of-plane electron current density in the electron inflow region. Such an electron current caused by $E_{z}$

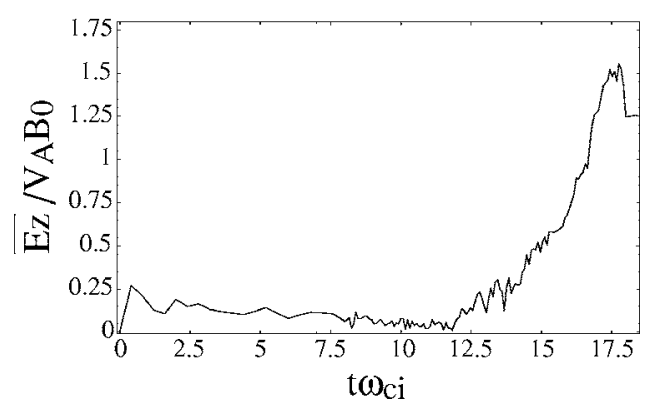

FIG. 5. Time evolution of the electric field directing toward the neutral sheet $\overline{E_{z}}=-z /|z| E_{z}$, averaged over the electron inflow region $\left(l_{x} / 2-0.2 \lambda_{i 0} \leq x\right.$ $\left.\leq l_{x} / 2+0.2 \lambda_{i 0}, 0.25 \lambda_{i 0} \leq|z| \leq 0.5 \lambda_{i 0}\right)$.

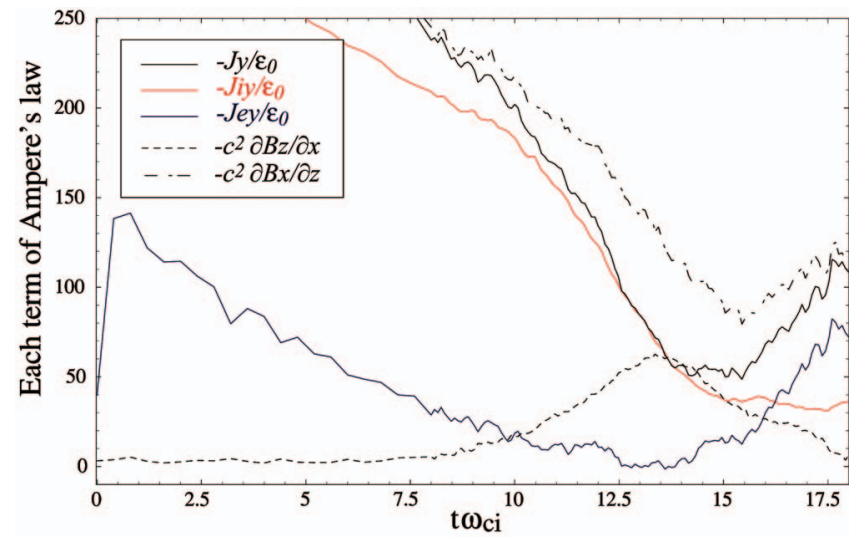

FIG. 6. (Color) Time history of each term on the right-hand side of Ampère's law $\left[\partial E_{y} / \partial t=-J_{y} / \varepsilon+c^{2}\left(\partial B_{x} / \partial z-\partial B_{z} / \partial x\right)\right]$ averaged over the electron inflow region. The black solid line denotes the contribution of the current density, which is calculated by summing up the ion and electron current densities (red and blue solid lines, respectively). The dashed and dashed-dotted lines represent the contribution from the rotation of the magnetic field.

also arises associated with the formation of the thin current sheet in the process of the convection driven in the nearEarth magnetotail, ${ }^{27}$ though the magnitude is much smaller than that associated with magnetic reconnection.

In order to see the role of the enhanced electron current in the electron inflow region, we plot in Fig. 6 the time evolution of each term on the right-hand side of Ampère's law, $\partial E_{y} / \partial t=-J_{y} / \varepsilon_{0}+c^{2}\left(\partial B_{x} / \partial z-\partial B_{z} / \partial x\right)$, averaged over the electron inflow region $\left(l_{x} / 2-0.2 \lambda_{i 0} \leq x \leq l_{x} / 2+0.2 \lambda_{i 0}\right.$, $\left.0.25 \lambda_{i 0} \leq|z| \leq 0.5 \lambda_{i 0}\right)$. The term contributed by the electron current (blue solid line) decreases until $t \omega_{c i} \approx 13.5$, which results from the convection of the initial current carriers away from the $\mathrm{X}$ line. After then, however, this term increases again due to the enhancement of the out-of-plane electron drift caused by the polarization electric field. The important point in this figure is that the contribution from the $x$ gradient of the magnetic field $B_{z}$ also has a peak at $t \omega_{c i}$ $\approx 13.5$. The absolute value of $\partial B_{z} / \partial x$ in the electron inflow region reflects a curvature of the magnetic field lines, which is expressed as $\kappa \simeq\left|B_{x} / B^{2} \partial B_{z} / \partial x\right|$ when $\left|B_{z} / B\right| \ll 1$, where $B=\sqrt{B_{x}^{2}+B_{z}^{2}}$. Since $B_{x}$ (not shown) is almost constant after $t \omega_{c i} \approx 14, \kappa$ is mostly proportional to $\left|\partial B_{z} / \partial x\right|$ at its declining phase. Therefore it is found that the curvature of the magnetic field lines decreases as the out-of-plane electron current in the electron inflow region is enhanced. This means that the magnetic field lines in the upstream region tends to be parallel to the $x$ direction, leading to the extension of the electron meandering region along the $x$ direction. Note that the left-hand side of Ampère's law ( $\partial E_{y} / \partial t$, not shown) is mostly zero within the present simulation time in comparison with each term on the right-hand side.

Figure 7 shows the snapshots of the out-of-plane electron velocity $V_{e y}$ with the magnetic field lines (white solid lines) around the $X$ line. The strong electron velocity seen near the center of the simulation area indicates that electrons are accelerated by $E_{y}$ and perform the meandering motions. Thus, this region is mostly interpreted as the electron diffusion region. As the curvature of the magnetic field lines de- 


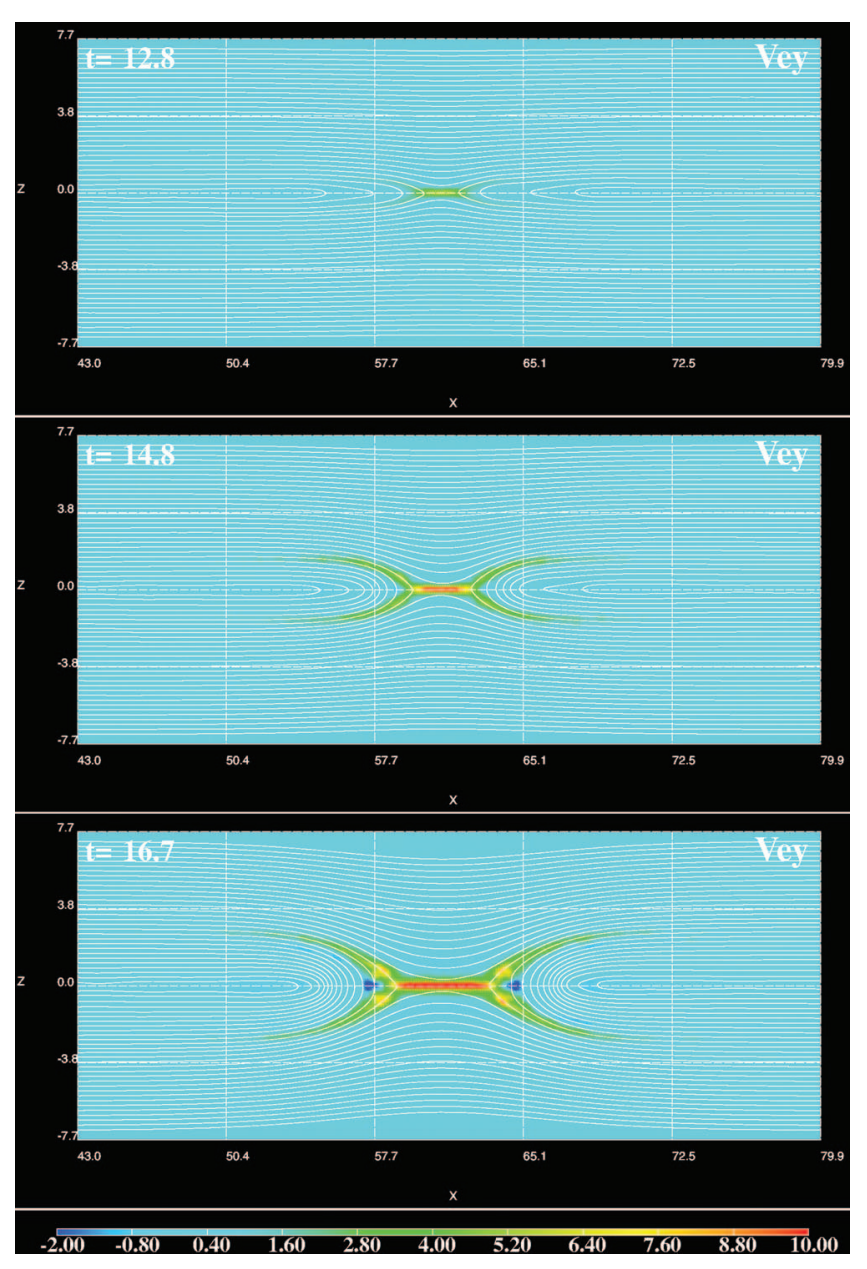

FIG. 7. (Color) Snapshots of the out-of-plane electron flow velocity at $t \omega_{c i}=12.8,14.8$, and 16.7. Magnetic field lines (white solid lines) are superposed on each panel.

creases in the electron inflow region, the electron meandering region, that is, the electron diffusion region tends to extend along the $x$ direction. This is also evident in Fig. 8, which describes time evolutions of (a) the length $l$, (b) the width $\delta$, and (c) the aspect ratio $\delta / l$ of the electron diffusion region. The length $l$ and the width $\delta$ of the electron diffusion region are properly determined from the simulation results at a given time. The electron diffusion region should be the region where the dissipation processes are mainly supported by the electron dynamics, that is, the region where electrons are accelerated by the inductive electric field $E_{y}$ and experience the meandering/Speiser motions. ${ }^{32}$ Thus, the downstream edge of the electron diffusion region is determined by the location where the electron outflow velocity along the $x$ axis reaches a peak value. Outside the edge, electrons are decelerated and the electron flow energy is partly converted to its thermal energy. On the other hand, the upstream edge of the electron diffusion region is determined by the turning point of the electron meandering motions. The amplitude of the electron meandering orbit is given by $\delta_{m}=\lambda_{g}\left(\delta_{m}\right)$, where $\lambda_{g}(z)=v_{\text {th }} / \omega_{c}$ is the local Larmor radius of electrons, $v_{\text {th }}$ $=\sqrt{2} T_{e} / m_{e}$ is the electron thermal velocity, and $\omega_{c}=$ $-e B_{x} / m_{e}$ is the electron cyclotron frequency. The width $\delta$ is defined by $\delta=\delta_{m}^{+}+\left(-\delta_{m}^{-}\right)$, where $\delta_{m}^{ \pm}$denotes the location of
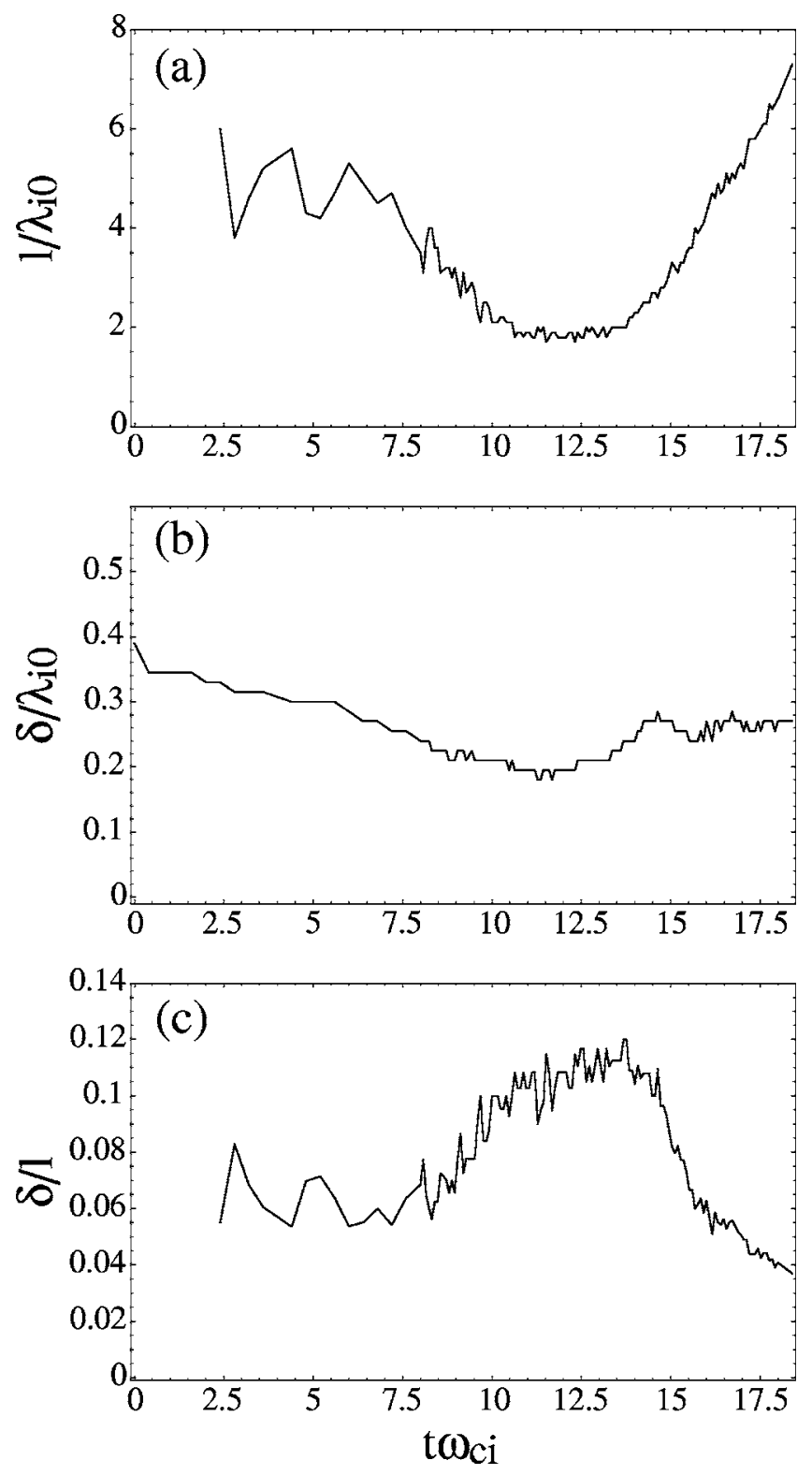

FIG. 8. Time evolutions of (a) the length, (b) the width, and (c) the aspect ratio of the electron diffusion region, respectively.

the upstream edge in the $\pm z$ region. The length $l$ of the electron diffusion region increases as time goes on after $t \omega_{c i}$ $\approx 13.5$ [Fig. 8(a)] when the curvature of the magnetic field lines reaches the peak. Since the width $\delta$ is mostly constant in time [Fig. 8(b)], the aspect ratio $\delta / l$ decreases after $t \omega_{c i}$ $\approx 13.5$, especially after $t \omega_{c i} \approx 15$, so that a long and narrow diffusion region is formed [Fig. 8(c)].

In Fig. 9, a schematic field structure around the electron diffusion region is described with the polarization electric field $E_{z}$ and the out-of-plane current density $J_{y}$. The electric field directing toward the neutral sheet is significant in the shadowed regions. The out-of-plane current density $J_{y}$ in the shadowed region is enhanced in the manner that the polarization electric field $E_{z}$ forces the inflow electrons to perform the $\boldsymbol{E} \times \boldsymbol{B}$ drift to the out-of-plane direction and enhances the out-of-plane electron current density. Since the out-of-plane current density is almost uniform along the upstream edge of 


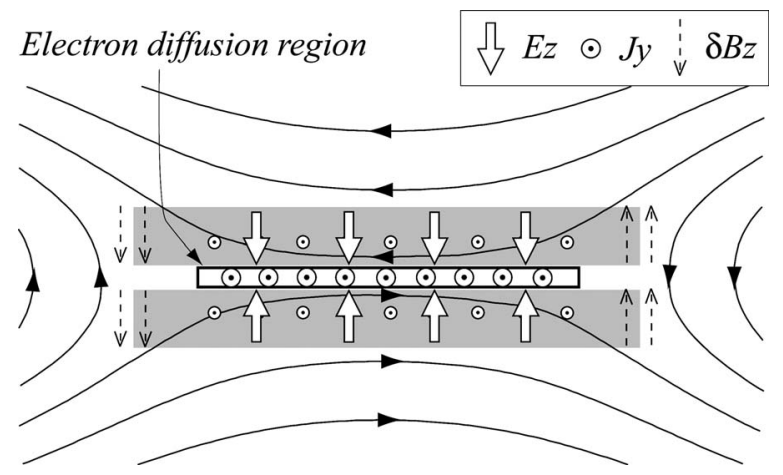

FIG. 9. Schematic field structure including the polarization electric field $E_{z}$ and out-of-plane current around the electron diffusion region. The electric field directing toward the neutral sheet is significant in the shadowed regions. The out-of-plane currents in the shadowed regions produce the magnetic field $\delta B_{z}$ (dashed arrows) at the edges, which reduces the curvature of the field lines in the electron inflow region and extends the electron diffusion region along the $x$ axis.

the electron diffusion region, the magnetic field $B_{z}$ does not appear near the $\mathrm{X}$ line. Thus the magnetic field lines in the electron inflow region should be parallel to the upstream edge, so that the curvature of the magnetic field lines is reduced in association with the enhancement of $J_{y}$ in the electron inflow region. On the other hand, near the downstream edge of the shadowed region, $J_{y}$ has a gradient along the $x$ direction, which gives rise to the magnetic field fluctuation $\delta B_{z}$ (dashed arrows in Fig. 9) and also reduce the curvature of the magnetic field lines. As a result, the magnetic field lines in the electron inflow region tend to be parallel to the $x$ axis, leading to the extension of the electron meandering region, that is, the electron diffusion region along the $x$ direction.

\section{Impact on the reconnection rate}

We consider here how the structure change of the electron diffusion region affects the reconnection rate, that is, the electric field along the $\mathrm{X}$ line. Figure 10 shows the flow

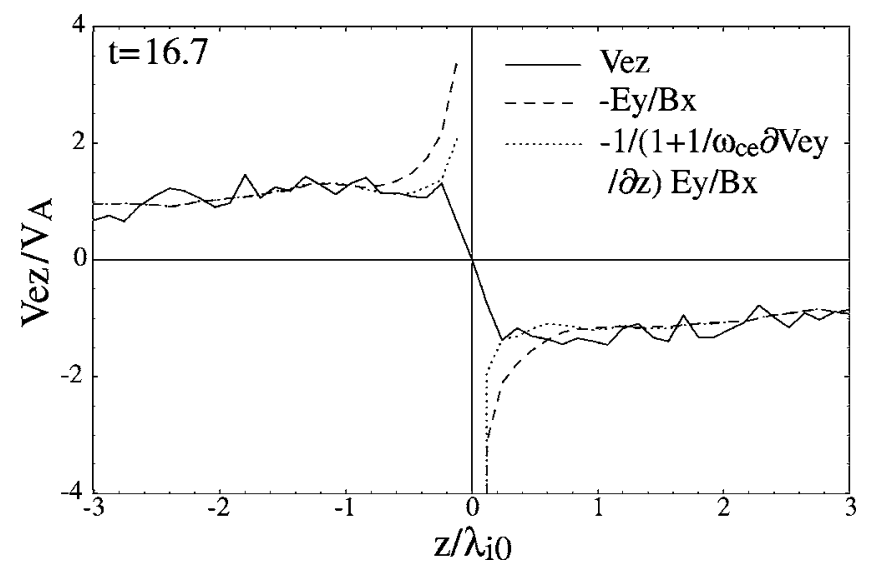

FIG. 10. Flow velocity of electrons (solid line) along the $z$ direction at $x$ $=l_{x} / 2$ and $t \omega_{c i}=16.7$. The dashed line denotes the $\boldsymbol{E} \times \boldsymbol{B}$ drift velocity defined by $-E_{y} / B_{x}$, and the dotted line is a velocity described by $-1 /(1$ $\left.+1 / \omega_{c e} \partial V_{e y} / \partial z\right) E_{y} / B_{x}$, which expresses the "modified $\boldsymbol{E} \times \boldsymbol{B}$ drift velocity" due to a large $E_{z}$. velocity of electrons along the $z$ direction at $x=l_{x} / 2$. This profile is useful to understand the electron behavior around the $\mathrm{X}$ line, when the intense polarization electric field is imposed. The dashed line expresses the $\boldsymbol{E} \times \boldsymbol{B}$ drift velocity in the $z$ direction, that is, $-E_{y} / B_{x}$. The $\boldsymbol{E} \times \boldsymbol{B}$ drift velocity diverges from the electron flow velocity at $z \approx \pm 0.7 \lambda_{i 0}$, so that the frozen-in condition for electrons breaks down in the region between $z \approx \pm 0.7 \lambda_{i 0}$. However, the upstream edges of the electron diffusion region where meandering electrons turn back to the neutral sheet is located at $z \approx \pm 0.15 \lambda_{i 0}$ in our definition. Actually, the strong polarization electric field imposed inside the ion-scale diffusion region makes the inertial term in the generalized Ohm's law (1) considerably large and suppress the electron inflow velocity. In this case, the generalized Ohm's law (1) can be expressed in the form

$$
E_{y}+V_{e z} B_{x} \simeq-\frac{m_{e}}{e} V_{e z} \frac{\partial V_{e y}}{\partial z},
$$

where $V_{e y} \simeq E_{z} / B_{x}$ is a good approximation [the $\left(m_{e} / e\right) \partial V_{e y} / \partial t$ term is negligible compared to the $E_{y}$ term (e.g., Ref. 19)]. Thus, we can obtain the electron inflow velocity,

$$
V_{e z} \simeq-\frac{1}{1+\omega_{c e}^{-1} \partial V_{e y} / \partial z} \frac{E_{y}}{B_{x}}
$$

where $\omega_{c e}$ is the local electron cyclotron frequency. We call this velocity the "modified $\boldsymbol{E} \times \boldsymbol{B}$ drift velocity." This profile is plotted by the dotted line in Fig. 10. The modified $\boldsymbol{E} \times \boldsymbol{B}$ drift velocity diverges from the electron inflow velocity at $z \approx \pm 0.15 \lambda_{i 0}$, which are consistent with the upstream edges of the electron diffusion region.

Assuming that the inflow electrons along the $z$ direction are mostly accelerated toward the $x$ direction by the magnetic tension in the electron diffusion region, we can obtain the approximation

$$
-m_{e} n_{e 1} V_{e 1} V_{e 2} \simeq \frac{B_{1} B_{2}}{\mu_{0}},
$$

where $n_{e j}, V_{e j}$, and $B_{j}$ are, respectively, the electron density, velocity, and magnetic field in the location denoted by $j$ ( $j$ $=1$ and 2 represent the upstream and downstream edges of the electron diffusion region, respectively), that is, $V_{e 1}$ $=V_{e z}\left(x=l_{x} / 2, z=\delta_{m}^{+}\right), \quad V_{e 2}=V_{e x}\left(x=l^{+}, z=0\right), \quad B_{1}=B_{x}(x$ $\left.=l_{x} / 2, z=\delta_{m}^{+}\right), B_{2}=B_{z}\left(x=l^{+}, z=0\right)$, and $l^{+}$is the location of the right-hand edge of the electron diffusion region. The outof-plane electric field $E_{y}$ is assumed to be spatially uniform around the electron diffusion region, so that

$$
E_{y} \simeq-V_{e 1}^{\prime} B_{1}^{\prime} \simeq-V_{e 1} B_{1}-\frac{m_{e}}{e} V_{e 1}\left(\frac{\partial V_{e y}}{\partial z}\right)_{1} \simeq V_{e 2} B_{2},
$$

where the prime denotes the location at which the electron inflow velocity diverges from the $\boldsymbol{E} \times \boldsymbol{B}$ drift velocity $\left(-E_{y} / B_{x}\right)$, that is, $V_{e 1}^{\prime}=V_{e z}\left(x=l_{x} / 2, z=0.7 \lambda_{i 0}\right)$, and $B_{1}^{\prime}=B_{x}(x$ $\left.=l_{x} / 2, z=0.7 \lambda_{i 0}\right)$ at $t \omega_{c i}=16.7$, for example. We further assume $V_{e 1}^{\prime} \simeq V_{e 1}$, which implies for the incompressible fluid that all the electrons entering the region where the frozen-in condition breaks down reach the electron diffusion region 


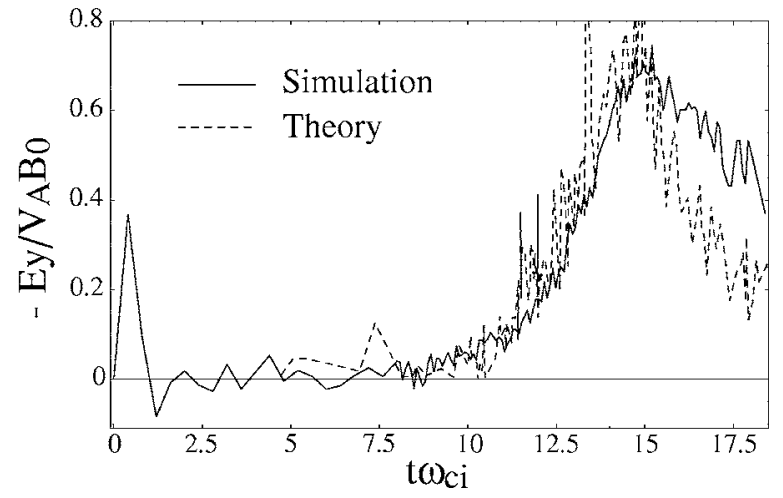

FIG. 11. A comparison of the time evolutions of the out-of-plane electric field. The solid line denotes the direct measurement of the simulation results at the center of the simulation area. The dashed line is the theoretical prediction calculated from the upstream parameters $\left[n_{e 1}, B_{1}\right.$, and $\left.\left(\partial V_{e y} / \partial z\right)_{1}\right]$ and the size of the electron diffusion region ( $\delta$ and $l$ ) determined by the simulation results.

defined in the present study. From (5) and (6) using $l\left|V_{e 1}\right|$ $\simeq \delta\left|V_{e 2}\right|$ for continuity, one can derive

$$
\begin{aligned}
& V_{e 2} \simeq-\sqrt{\frac{B_{1}^{\prime}}{B_{1}}} V_{\mathrm{A} e}, \\
& E_{y} \simeq-\frac{\delta}{l}\left[1+\frac{1}{\omega_{c e, 1}}\left(\frac{\partial V_{e y}}{\partial z}\right)_{1}\right]^{3 / 2} B_{1} V_{\mathrm{A} e},
\end{aligned}
$$

where $V_{\mathrm{A} e}=B_{1} / \sqrt{\mu_{0} n_{e 1} m_{e}}$ is called the electron Alfvén velocity. Since $B_{1}^{\prime} / B_{1} \gtrsim 1$, it is found that the electron outflow velocity can exceed the electron Alfvén velocity defined at the upstream edge of the electron diffusion region. The theoretical prediction of the out-of-plane electric field using (8) is plotted as a function of time in Fig. 11 (dashed line), superposed on the direct measurement from the simulation results (solid line). This theoretical prediction is calculated from the upstream parameters $\left[n_{e 1}, B_{1}\right.$, and $\left.\left(\partial V_{e y} / \partial z\right)_{1}\right]$ and the size of the electron diffusion region ( $\delta$ and $l$ ) determined by the simulation results at each given time. It increases as time goes on until it reaches a peak at $t \omega_{c i} \approx 15$ and then starts to drop associated with the reduction of the aspect ratio $(\delta / l)$, that is, with the extension of the electron diffusion region. This profile is consistent with the simulation results (solid line). The damping rate of $E_{y}$ after $t \omega_{c i} \approx 15$ is slightly different between the simulation results and the theoretical prediction. We think that this is caused by the underestimation of $\delta$. In our model, we assume that all the inflow electrons are accelerated toward the $x$ direction only in the electron diffusion region defined in the present study. However, the polarization electric field $E_{z}$ directing to the neutral sheet forces the inflow electrons toward the out-of-plane direction, even when they reside outside the electron diffusion region. Thus, the acceleration region should be wider along the $z$ direction than the electron meandering region, though it is difficult to correctly determine the effective value of $\delta$.

\section{Comparison with the case of $m_{i} / m_{e}=1$}

In order to emphasize the role of the polarization electric field caused by the ion meandering motions around the $\mathrm{X}$

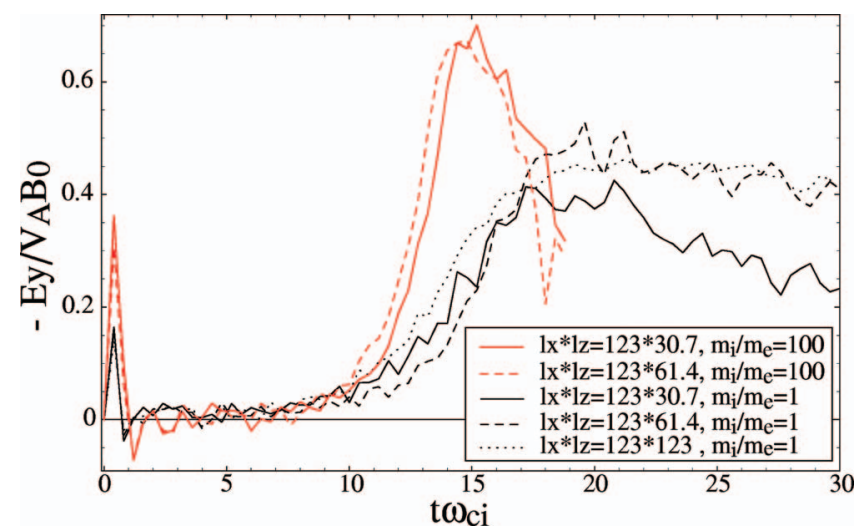

FIG. 12. (Color) A comparison of the time evolutions of the out-of-plane electric field at the center of the simulation area (the reconnection rate). Red lines denote the runs for $m_{i} / m_{e}=100$, and black lines the runs for $m_{i} / m_{e}$ $=1$. Two system sizes are examined under each mass ratio: $l_{x} \times l_{z}=123 \lambda_{i 0}$ $\times 30.7 \lambda_{i 0}$ (solid lines), and $123 \lambda_{i 0} \times 61.4 \lambda_{i 0}$ (dashed lines). A larger system size with $l_{x} \times l_{z}=123 \lambda_{i 0} \times 123 \lambda_{i 0}$ is also employed under $m_{i} / m_{e}=1$ (shown by a black dotted line).

line, we compare the results discussed in the previous sections with those obtained from the simulations under $m_{i} / m_{e}$ $=1$, in which the decoupling between ions and electrons does not arise, so that the polarization electric field is eliminated. At the same time, we check the effects of the conducting wall located at the boundary of the simulation area in the $z$ direction by performing the same simulations in a larger system in the $z$ direction. In the runs with $m_{i} / m_{e}=1$, the temperature ratio in the initial plasma sheet $\left(T_{i, \mathrm{ps}} / T_{e, \mathrm{ps}}\right)$ is also set as unity in order to avoid the electrostatic field arising from the difference in the Larmor radius between ions and electrons. The system parameters in the case of $m_{i} / m_{e}=1$ are $\Delta_{L_{B}}=0.96 \lambda_{i 0}, \Delta_{L_{D}}=0.12 \lambda_{i 0}, \Delta t \omega_{c i}=0.01$, and $c / V_{\mathrm{A}}=6.0$. The other conditions are the same as the case of $m_{i} / m_{e}=100$. We examine several system sizes: $l_{x} \times l_{z}=123 \lambda_{i 0} \times 30.7 \lambda_{i 0}$, and $123 \lambda_{i 0} \times 61.4 \lambda_{i 0}$ for the $m_{i} / m_{e}=100$ runs, and $l_{x} \times l_{z}$ $=123 \lambda_{i 0} \times 30.7 \lambda_{i 0}, 123 \lambda_{i 0} \times 61.4 \lambda_{i 0}$, and $123 \lambda_{i 0} \times 123 \lambda_{i 0}$ for the $m_{i} / m_{e}=1$ runs.

The time evolution of the out-of-plane electric field at the center of the simulation area (the reconnection rate) is shown in Fig. 12 for each run. In the case of $m_{i} / m_{e}=1$, we find that a quasi-steady reconnection is achieved in a larger system, although the reconnection rate in the smaller system $\left(l_{x} \times l_{z}=123 \lambda_{i 0} \times 30.7 \lambda_{i 0}\right)$ tends to drop due to the effects of the conducting wall boundary. On the other hand, in the case of $m_{i} / m_{e}=100$, the time evolution of the reconnection rate is independent of the system size, rising until $t \omega_{c i} \approx 15$ and then falling down associated with the extension of the electron diffusion region. These results demonstrate the importance of the polarization electric field $E_{z}$ in suppressing the reconnection processes. Another important point is that the asymptotic reconnection rate in the $m_{i} / m_{e}=1$ runs $\left(\left|E_{y}\right| \sim 0.45 V_{\mathrm{A}} B_{0}\right.$ $\left.=0.09 V_{\mathrm{A} 0} B_{0}\right)$ is large enough to explain fast dissipative events, even though the Hall effects are not included. Therefore it is suggested that the Hall effects should not be necessarily required for fast reconnection. This is well consistent with the results of Karimabadi et al. ${ }^{23}$ 


\section{DISCUSSION AND CONCLUSIONS}

In this paper, we have investigated time evolutions of the electron diffusion region embedded in the ion-scale diffusion region and the resulting reconnection rate associated with magnetic reconnection in a fully kinetic and large system. We performed 2-1/2 dimensional full particle simulations with the adaptive mesh refinement (AMR) technique and the particle splitting algorithm. ${ }^{24}$ The adoption of these techniques to the conventional particle-in-cell (PIC) code enables us to perform effectively high-resolution simulations, including kinetic processes of magnetic reconnection. Previous reconnection studies using full particle simulations in the periodic system ${ }^{17-20}$ achieved fast reconnection, but the system did not reach steady state and the reconnection rate decreased after it reached a peak value. The authors of the previous studies suggest that this is because the compressional effects in the magnetic islands affect the reconnection processes in small periodic systems. The full particle code with the AMR and particle splitting employed in the present study allows us to take a large system, in which the effects of the downstream periodic boundaries hardly affect the reconnection processes within the present simulation time.

It is found that the reconnection rate increases associated with magnetic reconnection and reaches a peak value large enough for fast reconnection, but then it decreases as time goes on, even though the periodicity of the system is negligible. The key process responsible for slowing magnetic reconnection is the extension of the electron diffusion region associated with the evolution of the strong polarization electric field $E_{z}$ imposed in the electron inflow region. The polarization electric field directing toward the neutral sheet is caused by the inertia difference between ions and electrons, and enhanced by the meandering motions of the background ions. Because the hot ions initially loaded in the Harris-type current sheet have a large velocity in the out-of-plane direction, they can easily escape from the diffusion region due to the Lorentz force before their meandering motions become dominant. Thus their impact on the strong $E_{z}$ is small. The polarization electric field $E_{z}$ forces the inflow electrons to move toward the out-of-plane direction by the $\boldsymbol{E} \times \boldsymbol{B}$ drift and enhances the out-of-plane current density $J_{y}$ in the electron inflow region. The role of $J_{y}$ is to reduce the curvature of the magnetic field lines so that they are mostly parallel to the $x$ direction near the $\mathrm{X}$ line, in such a way that $J_{y}$ becomes uniform along the upstream edge of the electron diffusion region. As a result, the electron meandering region, that is, the electron diffusion region, extends along the $x$ direction.

We performed a Sweet-Parker-like analysis around the electron diffusion region, taking into account the breakdown of the electron frozen-in condition in the electron inflow region, and demonstrate that the extension of the electron diffusion region can suppress the reconnection rate. In order to emphasize the role of the polarization electric field and the Hall effects, we compare the simulation runs with $m_{i} / m_{e}$ $=1$ and $m_{i} / m_{e}=100$. It is found that (1) a quasi-steady reconnection is achieved in the $m_{i} / m_{e}=1$ cases where the polarization electric field does not appear, different from the $m_{i} / m_{e}=100$ cases; (2) a large reconnection rate with $\left|E_{y}\right|$ $\sim 0.09 V_{\mathrm{A} 0} B_{0}$ is obtained in the $m_{i} / m_{e}=1$ runs, even though they do not include the Hall effects, so that the Hall effects are not necessarily required for fast reconnection.

The polarization electric field $E_{z}$ caused by the ion meandering motions has been seen in previous studies within large systems using hybrid simulations. ${ }^{21,29}$ However, their systems achieved a quasi-steady reconnection, different from the present results. This inconsistency is possibly attributed to the absence of the electron meandering effects in the previous simulations. The damping of the reconnection rate results from the extension of the electron diffusion region in which electrons carry out the meandering motions. The electron meandering effects should be incorporated into the term arising from the nongyrotropic electron pressure of the generalized Ohm's law [the second term on the right-hand side of (1)]. ${ }^{9-13}$ However, both of the previous studies did not include this term.

Full particle simulations of magnetic reconnection without the effects of the periodic boundaries have been performed by Pei et $a l .{ }^{6}$ under an open system. They also investigated a time evolution of the diffusion region and demonstrated that the system reached a steady-state reconnection and the structure was mostly unchanged during the simulation time. Magnetic reconnection in their system is driven by an external electric field, supplying an external plasma into the system. The reconnection rate in such a driven system is mainly controlled by the external driving electric field. ${ }^{6,33}$ Thus, the size of the diffusion region should be determined in the manner that the output of the plasma and magnetic field flux from the system becomes consistent with the input from the external region. Thus, the reconnection processes in the driven system are essentially different from those in the spontaneous system, as employed in the present study, in which there is no energy input from the external region.

The question that remains is what can support fast reconnection in actual space plasma after the reconnection rate reaches the peak. In fact, the peak value of the reconnection rate obtained in the present runs is $\sim 0.7 V_{\mathrm{A}} B_{0}$. If we take $B_{0} \sim 10 \mathrm{nT}$ and $n_{\mathrm{ps}} \sim 0.5 \mathrm{~cm}^{-3}$, we can calculate $\left|E_{y}\right|$ $\sim 2.2 \mathrm{mV} / \mathrm{m}$, which is not unrealistic compared with observational results. ${ }^{34}$ However, in the present model, the reconnection rate is expected to continue decreasing after the meandering motions due to the background cold ions have been dominant around the $\mathrm{X}$ line. Thus, it is supposed that other mechanisms that are not included in the model might play an important role in enhancing the reconnection rate. In the two-dimensional system employed in the present study, plasma instabilities excited along the $y$ direction are suppressed, in principle. Actually, since electrons in the electron diffusion region can be strongly accelerated toward the $y$ direction beyond the electron Alfvén velocity defined in the upstream region, a large velocity difference between ions and electrons is expected to arise. Figure 13 shows out-of-plane velocity distribution functions for ions (dashed line) and electrons (solid line) around the center of the simulation area. Both distributions are not simply Maxwellian. Ions have a weak beam component at $v_{y} \approx-1.0 V_{A}$, while electrons provide a long-tail structure in a high-energy part. 


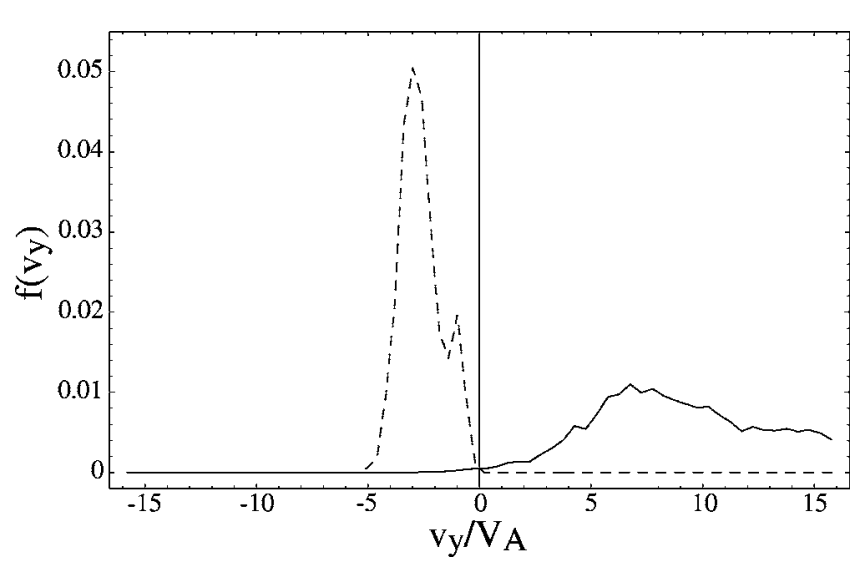

FIG. 13. Out-of-plane velocity distribution functions $f\left(v_{y}\right)$ for ions (dashed line) and electrons (solid line) around the center of the simulation area at $t \omega_{c i}=14.8$.

However, apart from these small components, the difference in the bulk velocity between ions and electrons is about $10 V_{\mathrm{A}}$, which is far beyond the electron thermal spread $\left(\approx 3.5 V_{\mathrm{A}}\right)$.Thus, the Buneman-type instability could quickly develop along the $y$ direction in three-dimensional systems. ${ }^{35}$ Indeed, such an instability and its nonlinear structure have been demonstrated in a three-dimensional system with a strong guide field, ${ }^{36}$ and can give rise to the anomalous resistivity, so that the reconnection rate $\left(\left|E_{y}\right|\right)$ is enhanced. It is suggested that the Buneman-type instability can be suppressed due to an electron self-heating within the electron diffusion region in a system without the guide field. ${ }^{37}$ The heating mechanism is simple. The total flow energy into the electron diffusion region is converted to the total thermal energy via the meandering motions near the $\mathrm{X}$ line. In the present simulation, however, the initial thermal velocity of electrons $\left(v_{\text {th }, e}=3.3 V_{\mathrm{A}}\right)$ is beyond the inflow velocity $\left(V_{e 1}\right.$ $\approx 1.2 V_{\mathrm{A}}$, see Fig. 10), so that the electron self-heating does not occur in the present setup. In order to investigate the role of the Buneman-type instability in the electron diffusion region, it is necessary to perform a large-scale kinetic simulation in a three-dimensional system.

\section{ACKNOWLEDGMENTS}

The author would like to thank S. Machida for useful and informative discussions. The simulations are performed by FUJITSU HPC2500 installed at the Academic Center for Computing and Media Studies of Kyoto University, and the Information Technology Center of Nagoya University. This work has been partly supported by the Grant-in-Aid for JSPS Fellows.

${ }^{1}$ V. M. Vasyliunas, Rev. Geophys. 13, 303 (1975).

${ }^{2}$ P. A. Sweet, in Electromagnetic Phenomena in Cosmical Physics, edited by B. Lehnert (Cambridge University Press, London, 1958), p. 123.

${ }^{3}$ E. N. Parker, Astrophys. J., Suppl. Ser. 8, 177 (1963).

${ }^{4}$ H. E. Petschek, in AAS-NASA Symposium on the Physics of Solar Flares, NASA Spec. Publ., SP-50, 1964, p. 425.

${ }^{5}$ L. Spitzer, Jr., Physics of Fully Ionized Gases, 2nd ed. (Interscience, New York, 1962), p. 139.

${ }^{6}$ W. Pei, R. Horiuchi, and T. Sato, Phys. Rev. Lett. 87, 235003 (2001).

${ }^{7}$ B. U. Ö. Sonnerup, in Solar System Plasma Physics, edited by L. J. Lanzerotti, C. F. Kennel, and E. N. Parker (North-Holland, Amsterdam, 1979), Vol. III, p. 45.

${ }^{8}$ T. Terasawa, Geophys. Res. Lett. 10, 475 (1983).

${ }^{9}$ H. J. Cai and L. C. Lee, Phys. Plasmas 4, 509 (1997).

${ }^{10}$ M. Hesse and D. Winske, J. Geophys. Res. 103, 26,479 (1998).

${ }^{11}$ M. M. Kuznetsova, M. Hesse, and D. Winske, J. Geophys. Res. 103, 199 (1998).

${ }^{12}$ M. Hesse, K. Schindler, J. Birn, and M. Kuznetsova, Phys. Plasmas 6, 1781 (1999).

${ }^{13}$ M. Swisdak, J. F. Drake, M. A. Shay, and J. G. McIlhargey, J. Geophys. Res. 110, A05210 (2005).

${ }^{14}$ D. Biskamp, E. Schwarz, and J. F. Drake, Phys. Plasmas 4, 1002 (1997).

${ }^{15}$ J. Birn, J. F. Drake, M. A. Shay, B. N. Rogers, R. E. Denton, M. Hesse, M. Kuznetsova, Z. W. Ma, A. Bhattacharjee, A. Otto, and P. L. Pritchett, J. Geophys. Res. 106, 3715 (2001).

${ }^{16}$ J. D. Huba and L. I. Rudakov, Phys. Rev. Lett. 93, 175003 (2004).

${ }^{17}$ M. A. Shay, J. F. Drake, and B. N. Rogers, J. Geophys. Res. 106, 3759 (2001).

${ }^{18}$ M. Hesse, J. Birn, and M. Kuznetsova, J. Geophys. Res. 106, 3721 (2001).

${ }^{19}$ P. L. Pritchett, J. Geophys. Res. 106, 3783 (2001a).

${ }^{20}$ M. M. Kuznetsova, M. Hesse, and D. Winske, J. Geophys. Res. 106, 3799 (2001).

${ }^{21}$ M. A. Shay, J. F. Drake, B. N. Rogers, and R. E. Denton, Geophys. Res. Lett. 26, 2163 (1999).

${ }^{22}$ M. A. Shay, J. F. Drake, M. Swisdak, and B. N. Rogers, Phys. Plasmas 11, 2199 (2004).

${ }^{23}$ H. Karimabadi, D. Krauss-Varban, J. D. Huba, and H. X. Vu, J. Geophys. Res. 109, A09205 (2004).

${ }^{24}$ K. Fujimoto and S. Machida, J. Comput. Phys. 214, 550 (2006).

${ }^{25}$ G. Lapenta, J. Comput. Phys. 181, 317 (2002).

${ }^{26}$ A. M. Khokhlov, J. Comput. Phys. 143, 519 (1998).

${ }^{27}$ P. L. Pritchett and F. V. Coroniti, J. Geophys. Res. 100, 23551 (1995).

${ }^{28}$ M. Hesse, D. Winske, M. Kuznetsova, J. Birn, and K. Schindler, J. Geomagn. Geoelectr. 48, 749 (1996).

${ }^{29}$ K. Arzner and M. Scholer, J. Geophys. Res. 106, 3827 (2001).

${ }^{30}$ P. L. Pritchett, J. Geophys. Res. 106, 25961 (2001b).

${ }^{31}$ M. Hoshino, T. Mukai, T. Terasawa, and I. Shinohara, J. Geophys. Res. 106, 25979 (2001).

${ }^{32}$ T. W. Speiser, J. Geophys. Res. 70, 4219 (1965).

${ }^{33}$ T. Sato and T. Hayashi, Phys. Fluids 22, 1189 (1979).

${ }^{34}$ Y. Asano, T. Mukai, M. Hoshino, Y. Saito, H. Hayakawa, and T. Nagai, J. Geophys. Res. 109, A02212 (2004).

${ }^{35}$ O. Buneman, Phys. Rev. 115, 503 (1959).

${ }^{36}$ J. F. Drake, M. Swisdak, C. Cattell, M. Shay, B. Rogers, and A. Zeiler, Science 299, 873 (2003).

${ }^{37}$ A. Zeiler, D. Biskamp, J. F. Drake, B. N. Rogers, M. A. Shay, and M. Scholer, J. Geophys. Res. 107, 1230 (2002). 